Recepción: 18 / 03 / 2018

Aceptación: 20 / 05 / 2018

Publicación: 01/ 08 / 2018

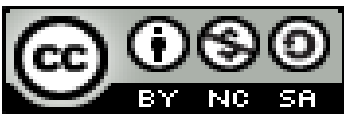

Ciencias de la salud

Artículo de investigación

\title{
Prevención del consumo de drogas en los estudiantes de la Facultad de \\ Ciencias Médicas
}

\section{Prevention of drug use in the students of the faculty of medical sciences}

\section{Prevenção do uso de drogas entre estudantes da Faculdade de Ciências Médicas}

\author{
Carmen Sánchez-Hernández ${ }^{\mathrm{I}}$ \\ carmen.sanchezh@ug.edu.ec \\ José Benalcazar-Gáme II \\ jose.benalcazag@ug.edu.ec \\ Delia Crespo-Antepara ${ }^{\mathrm{III}}$ \\ delia.crespoa@ug.edu.ec
}

\section{Correspondencia: carmen.sanchezh@ug.edu.ec}

\footnotetext{
${ }^{\text {I }}$ Universidad de Guayaquil, Docente, Directora de la Carrera de Enfermería, Especialista en Salud Comunitaria, Especialista en Gestión y Liderazgo.

II Universidad de Guayaquil, Docente, Vicedecano de la Facultad de Ciencias Médicas, Magister en Diseño Curricular.

III Universidad de Guayaquil, Docente, Directora de la Carrera de Obstetricia, Magister en gerencia clínica, salud sexual y reproductiva.
} 


\title{
Resumen
}

El siguiente trabajo de investigación tuvo como objetivo, investigar si se están tomando las medidas preventivas adecuadas para evitar el consumo de drogas en los estudiantes de la facultad de ciencias médicas 2016 - 2017. El tipo de estudio fue descriptivo, observacional, bibliográfico con enfoque cuantitativo. La población estuvo conformada por 90 estudiantes de La facultad de ciencias médicas y la muestra fue el total de la población. Se utilizó la técnica de la encuesta para la aplicación de dos instrumentos, una ficha de observación y un cuestionario. El análisis e interpretación de la información se realizó a través de la estadística descriptiva, en la que se logró recabar datos relevantes sobre la prevención y consumo de drogas; donde los resultados demostraron que el 50\% de los estudiantes encuestados consideren que si existió información y educación por parte de docentes sobre la prevención de consumo de drogas, mientras que el otro $50 \%$ de los estudiantes consideran que han sufrido algún tipo de discriminación por parte de amigos por no consumir drogas.

Palabras clave: prevención; consumo; drogas y riesgo.

\begin{abstract}
Prevention of the consumption of drugs in the students of the Faculty of Sciences Medical abstract the following research work aimed, investigate if appropriate preventive measures are being taken to prevent the consumption of drugs in students Faculty of medical sciences 20162017. The study was observational, descriptive, and bibliographical with quantitative approach. The population was comprised of 90 students of the Faculty of medical sciences and the sample was the total population. The survey technique was used for the application of the two instruments, an observation sheet and a questionnaire. The analysis and interpretation of information was carried out through the descriptive statistic, which was achieved to collect relevant data on prevention and drug use; where results showed that $50 \%$ of the students surveyed consider that if it existed information and education for teachers on the prevention of drug abuse, while another $50 \%$ of students believe that they have suffered some form of discrimination by friends by do not use drugs.
\end{abstract}

Keywords: prevention; consumption; drugs; risk. 


\section{Resumo}

A pesquisa seguinte teve como objetivo investigar se eles estão tomando as medidas adequadas para evitar o uso de drogas entre estudantes da Faculdade de Ciências Médicas medidas preventivas de 2016 - 2017. O tipo de estudo foi descritivo, observacional, literatura abordagem quantitativa. A população foi composta por 90 estudantes da Faculdade de Ciências Médicas e da amostra foi a população total. a técnica de pesquisa para a aplicação de dois instrumentos, foi utilizado um formulário de observação e um questionário. Análise e interpretação dos dados foi realizada através da estatística descritiva, nas quais é possível recolher dados relevantes sobre prevenção e uso de drogas; onde os resultados mostraram que 50\% dos alunos pesquisados consideram que se houvesse informação e educação por professores sobre a prevenção do uso de drogas, enquanto os outros $50 \%$ dos estudantes acreditam ter experimentado alguma forma de discriminação por amigos para não usar drogas.

Palavras chave: prevenção; consumo de; drogas e risco.

\section{Introducción}

Una de las problemáticas que enfrenta la sociedad es las drogas y Ecuador no escapa de esta "epidemia", donde pequeñas comunidades de los países se ven inmersas en esa problemática, por lo tanto, los docentes como involucrados en la labor social deben ejercer una acción preventiva, ya que no existe una fórmula mágica para acabar con las drogas. La prevención es La medida más efectiva que se puede tomar. Por ende, esta prevención exige un trabajo duro y constante por parte de todos, a fin de desarrollar en los estudiantes capacidades para evitar que caigan en el vicio de las drogas. El consumo de la droga se presenta en la actualidad como uno de los principales problemas que afronta la sociedad tanto por la magnitud del fenómeno como por las consecuencias del mismo. Es especialmente preocupante el uso indiscriminado de sustancias en periodos como la adolescencia etapa en la cual se ha incrementado en gran medida. Además, es necesario recordar que en este momento evolutivo es un periodo de transición de la infancia a la madurez en el que se producen una gran cantidad de cambios corporales conectivos, afectivos y de valores.

En Ecuador la edad promedio de inicio del consumo de drogas fue de 14 años, hasta el 2015, cuando en el 2010 era de 15 a 16 años, según un artículo que se encontró en el Diario Ecuador 
Inmediato, denominado CONSEP dio a conocer las cifras acerca del consumo de drogas, el cual también incluye el consumo de drogas ilícitas como el alcohol, tabaco y cigarrillo, estudio que tuvo como delimitación geográfica las principales ciudades del país como Quito y Guayaquil. (Ecuador Inmediato, 2017). La OMS público en su último reporte que el promedio capital anual de consumo de alcohol en el Ecuador fue igual a 7,2 litros anuales, de los cuales $90 \%$ son varones y 2,5\% pertenecen a los grupos de edad de los adolescentes menores de 18 años. La prevalencia de las drogas ilícitas es igual al $57 \%$ del cannabis, mientras que el $27 \%$ son consumidores de droga "H" o heroína y de cocaína. (Organización Panamericana de la Salud, 2014). Un artículo de Diario El Universo establece que el 71\% de los consumidores consume alcohol en las fiestas y reuniones de las amistades o de los propios familiares, mientras que un $12 \%$ afirma que adquiere los estupefacientes en el barrio, por medio de un amigo, así como también en los alrededores del plantel educativo. (Diario El Universo, 2014). La ciudad de Guayaquil concentra a más la tercera parte de los consumidores, donde a pesar que la marihuana continúa situándose en el primer lugar del ranking de las drogas más consumidas por los adictos y consumidores, sin embargo, la " $\mathrm{H}$ " es la de mayor crecimiento, especialmente en la población que se encuentra entre los 12 a los 18 años de edad, cuyos factores de riesgos pueden ser sociales, económicos, biológicos y psicológicos.

Con base a lo expuesto, en el presente artículo se estudiaron los factores de riesgos biopsicosociales y económicos asociados al consumo de drogas, considerando como delimitación geográfica espacial la Facultad de Ciencias Médicas de la Universidad de Guayaquil, que se encuentra formada por algunas Escuelas o Carreras, como es el caso de Enfermería, Tecnología Médica, Medicina y Obstetricia, cuyos estudiantes serán analizados a través de una indagación empírica para determinar el nivel del riesgo al que se encuentran expuestos los jóvenes que se educan en estas unidades académicas.

\section{Desarrollo}

\section{Consumo de drogas}

Los factores de riesgos que incrementan la probabilidad de que un individuo consuma drogas y que en el futuro inmediato se convierta en un adicto, son de tipo social como es el caso de la violencia en el hogar y la carencia afectiva, debido a padres migrantes y por lo tanto ausentes, que puede incidir en la depresión y en la búsqueda de la droga como solución a esta carencia, 
aunque también puede estar asociada a un factor genético o antecedente familiar, a la curiosidad o a un deseo de no ser rechazado por el grupo de amigos que ya consume sustancias psicoactivas. (Milián, Gálvez, Pita, y Rosales, 2014).

La droga constituye una de las sustancias químicas tóxicas que pueden encontrarse asociadas a un sinnúmero de enfermedades, desde leves irritaciones de las vías respiratorias, hasta afecciones crónicas que pueden implicar inclusive el aumento de las tasas de mortalidad por patologías como el cáncer, por ejemplo, especialmente hepático y pulmonar.

Según, (Rimassa, 2014) establece que "las drogas son sustancias psicoactivas no aptas para el consumo humano, ya que estas alteran la función de los organismos y sobre todo el sistema nervioso central, la administración puede ser por varias vías”. (pág. 2)

Por su parte, los adolescentes que consumen los diferentes tipos de drogas con mayor frecuencia son llamados adictos, debido a que este tipo de sustancias denominada con el apelativo de psicoactivas, provocan alteraciones graves en el sistema nervioso central y en ocasiones acelera o reduce las actividades de los órganos del cuerpo humano.

El (Instituto Nacional sobre Abuso de Drogas, 2012) expresa que "la droga puede ser natural o sintéticas, que al ser administrada o ingerida este actúa acelerando ciertas actividades físicas y mentales, ya que esta sustancia actúa directamente en el sistema nervioso”. (pág. 14). No todas las drogas actualmente se encuentran legalizadas dentro del país, aunque el cigarrillos, el tabaco, el alcohol sí tienen un componente jurídico que las hace legales, a pesar de ello no es recomendado el consumo de los mismos por parte del personal de salud, quienes en sus diferentes actividades referidas al tratamiento, recuperación, entre otras, prohíben el uso de las drogas, sin embargo por la fácil comercialización de estas sustancias, son adquiridas frecuentemente por los individuos.

\section{Tipos de Drogas}

La característica principal de las drogas es modificar el comportamiento de las personas que la consumen y su elección va de acuerdo a las necesidades del consumidor, entre las alteraciones que se pueden presentar incluyen síntomas sedantes, estimulantes, alucinógenos y afrodisíacos ocasionando un efecto en su salud mental y generando un alto riesgo de adicción. Según, (UNODC, Informe Mundial sobre las Drogas, 2016) establece que "las drogas están incluido en 
un grupo variado de sustancias, donde el potencial adictivo, es peligroso y este puede variar depende del consumo. Sin embargo, las características de los diferentes tipos son de elevada mortalidad”. (pág. 13).

Al respecto (Escohotado, 2014) difiere que los tipos de drogas con mayor consumo son las siguientes:

Alcohol. - Este consta de la fermentación de frutos y plantas, los efectos que provoca son agresión, depresión, adicción y muerte.

Heroína. - Su administración es de forma inyectada, los efectos que provoca esta sustancia es la falta de respiración, depresión, infecciones, riesgo de contraer VIH y Hepatitis.

Cocaína. - es una sustancia estimulante de color blanco, que son extraída de las hojas de coca y este es inhalado por la nariz.

Marihuana. - Esta droga es la más consumida, sus efectos son ataques de ansiedad, problemas al hablar y prestar atención. (pág. 1).

Al referirse a los tipos de drogas se indican a las diferentes categorías que se encuentran presentes como sedantes, estimulantes, alucinógenas y afrodisiacos, algunas de ellas son herbales procedentes de una planta, otras sintéticas o realizadas por el hombre utilizando sustancias químicas mezclando productos herbales y sintéticos.

\section{Epidemiología de la drogadicción}

La importancia de esta problemática actual social, psicológica es de mayor relevancia en los diferentes sistemas de salud público, actualmente es considerado como epidemia donde afecta a la mayor parte de adolescentes.

Al respecto (UNODC, 2016) expresa que "aproximadamente 246 millones de personas, expresan que el 5\% consumieron drogas ilícitas en una edad de 15 a 64 años durante el 2013, mientras que los hombres son más propensos que las mujeres al consumir marihuana, anfetaminas y cocaína, sin embrago las mujeres abusan de opioides, tranquilizantes".

El consumo de sustancias psicoactivas, ha sido una de las problemáticas a nivel mundial ya que se estima que 5 de cada 100 son consumidores de varias sustancia psicoactiva y esta a su vez la 
población es menor de 20 años de edad, la situación actual en el país radica en que no existe una prevención por parte de los gobiernos centrales y establecimientos de salud.

La epidemiologia de estas sustancias psicoactivas, afectan con gran magnitud a los jóvenes de la población mundial, ya sea este un país desarrollado o no, además la preocupación radica en las consecuencias por el consumo excesivo ya que este provoca alteraciones en el sistema nervioso central, así como también afecta a otros organismos del cuerpo, con lo cual con lleva a la muerte del individuo.

\section{Signos y síntomas del consumo de drogas}

Los individuos que consumen substancias psicotrópicas pueden presentar diferentes signos y síntomas de acuerdo al tipo de droga que consumen, sin embargo, algunas características que se presentan en forma general que hace uso de las drogas son el cambio repentino del comportamiento, cambios de humor, distanciamiento de los miembros de la familia, descuido de la apariencia personal y la pérdida de interés de las actividades favoritas.

Al respecto, el (Instituto de Neurociencias, 2015) expresa que dentro de los signos y síntomas de las drogas son las siguientes:

"Ojos inyectados de sangre.

El aliento es inusual.

Las pupilas grandes o pequeñas.

Aumento de apetito.

La pronunciación es lenta”. (pág. 4).

De acuerdo a la droga que consume el individuo se pueden presentar diferentes manifestaciones de comportamiento y en el aspecto físico, el consumo de cocaína puede ocasionar confusión, ansiedad, desconfianza, dilatación de las pupilas, pérdida del apetito, inquietud, irritabilidad, otra droga que es consumida con gran demanda es la marihuana la misma que presenta manifestaciones como ojos rojos, resequedad de la boca, pérdida de la conciencia, alucinaciones, desmotivación y cambios de humor.

Según, (Barros, 2013) manifiesta que los síntomas pueden ser físicos o emocionales:

"Pérdida de peso.

Mal rendimiento académico.

Actividades secretas. 
Robo.

Vómitos.

Pérdida de apetito". (pág. 2).

Los signos y síntomas que se pueden presentar son emocionales y sociales (mal humor, depresión, desmotivación, distanciamiento de las personas), los signos físicos (somnolencia, temblor, pérdida de peso, aumento del apetito, ojeras), signos psicológicos (no logran la concentración, miedo o preocupación, actitud defensiva), además de otros signos adicionales como pérdida del control, adoptan medidas extremas como el hurto y venta de accesorios de valor que generan un problema social que es la delincuencia.

\section{Factores de riesgos para el consumo de droga}

A continuación de los factores de riesgos y protección que influyen en el consumo de drogas es un fenómeno complejo donde intervienen los aspectos biológicos, psicológicos y sociales, para ello se analiza teóricamente cuales son las consecuencias del consumo excesivo de estas sustancias psicoactivas en los estudiantes:

\section{Factores riesgo biológico}

Al respecto existen otros factores que incluyen la etapa de desarrollo y condiciones médicas, por lo tanto, los jóvenes que padecen de trastornos mentales se ve afectados, ya que el riesgo seria mayor.

\section{(Hidalgo, Redondo, Barrio, \& Huertas, 2013), expresan lo siguiente:}

Dentro de los factores de riesgo biológicos el sexo masculino es la persona con mayor abuso de drogas, mientras que las mujeres al inicio asincrónico de la pubertad presentan baja autoestima y conducta riesgosa, el inicio del consumo empieza con la marihuana en una edad de 18 a 20 años. (pág. 140).

Los factores de biológicos son características de condiciones individuales y hábitos. Por lo tanto, la prevención en los jóvenes consumidores está relacionada con la capacidad de coordinar y aplicar estrategias para minimizar los riesgos y la mortalidad.

Al respecto, los autores (López, Rodríguez, \& Arias, 2013) establecen que "los factores de riesgos biológicos pueden poseer antecedentes hereditarios, aceptación por parte organismo, así como también el funcionamiento de bioquímicos-cerebral, por lo tanto, los efectos de los factores 
ambientales y la expresión de los genes de una persona se ven incluidos”. (p. 9). La prevención de los factores de riesgos en el consumo de droga trata de provenir el mayor número de riesgo y de defensa, dentro de esta problemática se analiza las variables tales como: el sexo, la edad, social, entre otros. Para ello la implementación de un programa preventivo debe contar con personal capacitado en la cual se puedan dirigir hacia la comunidad afectada.

\section{Factores riesgo psicológico}

Las psiquis en los jóvenes provocan la adquisición de sustancias psicoactivas constantemente, por lo tanto, la prevención por parte de los familiares debe ser importante, ya que se informará a sus hijos de las causas y consecuencias que este estupefaciente provoca en el organismo y en su sistema nervioso central.

Los autores (Secades y Fernández, 2012) establecen que:

Los factores psicológicos en los estudiantes buscan emociones nuevas, por lo cual el consumo de drogas es más alto, así como también la inteligencia baja, la agresividad enmarca la conducta antisocial por el uso de estas sustancias, además se asocian factores como la baja autoestima, inmadurez, impulsividad y rebeldía. (pág. 5).

Al respecto, del riesgo psicológico el ser humano busca emociones diferentes a lo vivido, por ende, los consumos de estas sustancias hacen se sienten libres de problemas, sin embargo, el uso excesivo de drogas contrae dificultades en sus sistemas y órganos internos debido a que estos químicos actúan creando un mal hábito y como consecuencia la mortalidad del mismo.

La (Enciclopedia de la Familia., 2011) manifiesta que el consumo y la adquisición de drogas "se da por curiosidad, ignorancia, presión, soledad, el joven al consumir esta sustancia química encuentra alivio a los problemas cotidianos, por ende, existen probabilidades de que este se vuelva adicto". (pág. 17). Por lo tanto, en la etapa en los que los jóvenes propios de su edad experimentan nuevas cosas, hacen que estos atraviesen por diversos cambios psicológicos y ven al mundo de otra manera, la inmadurez de la persona hace que este sea débil y de inicio al consumo volviéndose este cotidiano, por ende, se transformándose en adición.

\section{Factores riesgo económico}

Este factor fue analizado por expertos ya que la relación con la economía de los adictos, se dice que para ser consumidor este debe contar con ingresos económicos, ya que estas drogas tienen un 
precio elevado y adquirirlo de manera habitual genera gastos dentro del núcleo familiar. De acuerdo, a la publicado por la (Dirección de Salud Pública y Adicciones del Departamento de Salud del Gobierno Vasco y el SIIS Centro de Documentación y Estudios de la Fundación Eguía Careaga., 2014) manifiesta que "los factores que con lleva al consumo de sustancias estupefaciente "drogas" se da en muchas ocasiones por el nivel de ingresos que tiene la familia, por lo tanto, la adicción de los jóvenes tiene más probabilidades de dar inicio y mantenerlo por largos periodos". (p. 17)

Si bien es cierto, la economía es un elemento importante para dar inicio al consumo, en este caso la variedad de droga que existe y que pueden ser ingerido, consumido e inyectados el consumidor o adicto debe contar con estos recursos, por otro lado, si no posee dinero este se puede convertir en pordiosero o delincuente ya que la necesidad de conseguir la sustancia es inevitable.

Según, (Whiteford, 2013) expresa que "se permite demostrar que el índice de influencia en el consumo se da en personas que mantiene un ingreso económico estable, la frecuencia de ingerir esta sustancia psicotrópica provoca graves daños en la salud”. (p. 1). Mediante los criterios de estos expertos se debe considerar que el dinero está vinculado con el consumo de drogas, sin embargo, los jóvenes que no poseen con este factor se convierten en delincuentes, ya que se vuelven adictos y el abandono de estas sustancias es difícil.

\section{Prevención sanitaria del consumo de droga}

La prevención del consumo de varios tipos de drogas dentro de la etapa educativa, engloba los contenidos de la educación para la salud, este a su vez es un tema de suma importancia y obligatoria de los profesionales sanitarios para facilitar los cambios de conducta de los pacientes. De acuerdo, (Asensio, Zarco, Villanueva, y Caudevilla, 2012) manifiestan lo siguiente:

Es importante destacar que los aspectos preventivos mantienen varios tipos de incorporación, en las cuales se deben emplear proyectos en los centros educativos, curricular y programación en Aula. Para esto el desarrollo de campañas, entregas de volantes y otras actividades son estrategias que permite que los estudiantes doten por herramientas adecuadas y puedan tomar decisiones sobre su salud y para las personas que lo rodean. (p.33) 
Al respecto los profesionales sanitarios deben capacitar con material informativo sobre la variedad de droga que existe, así como también los padres de familia puedan contribuir con información básica, los problemas que estos ocasionan al ser un consumidor.

\section{Metodología}

La metodología de estudio fue de carácter cuantitativo y su tipología descriptiva y de campo. La descripción permitió precisar la problemática del estudio referente a los factores de riesgos biopsicosociales y económicos asociados al consumo de drogas, permitiendo obtener información numérica y porcentual de la identificación de los estudiantes de la Facultad de Ciencias Médicas, además fue transversal porque se realizó en el tiempo presente considerando un periodo de estudio desde septiembre del 2016 hasta febrero del 2017. La población involucrada fueron 1.350 estudiantes de la Facultad de Ciencias Médicas, de las diferentes Escuelas de Medicina, Enfermería, Obstetricia y Tecnológica Médica, y la muestra 90 estudiantes considerados de forma aleatoria. Se utilizó la técnica de la encuesta para la obtención de la información que permitió conocer las causas psico-social del núcleo familiar, identificando los riesgos físicos y psíquicos por el consumo de drogas, las causas que lleva a los adolescentes a consumir drogas y los factores de riesgo o complicaciones por consumo de drogas.

\section{Resultados}

El análisis de los datos obtenidos posterior a la aplicación del instrumento se presenta a continuación:

Tabla1. Análisis frecuencial y porcentual del consumo de drogas en los estudiantes de la facultad de ciencias médicas.

\begin{tabular}{|l|l|l|l|l|l|l|l|}
\hline ITEMS & PERSONAS & \multicolumn{2}{l|}{ SIEMPRE } & \multicolumn{2}{l|}{ A VECES } & \multicolumn{2}{l|}{ NUNCA } \\
\hline $\begin{array}{l}\text { 1.- ¿Con qué frecuencia usted ha } \\
\text { sabido si algún familiar suyo } \\
\text { consume o consumió drogas? }\end{array}$ & 90 & $\begin{array}{l}\text { TOTA } \\
\text { L }\end{array}$ & $\%$ & $\begin{array}{l}\text { TOTA } \\
\text { L }\end{array}$ & $\%$ & $\begin{array}{l}\text { TOTA } \\
\text { L }\end{array}$ & $\%$ \\
\cline { 2 - 8 } & 10 & $11 \%$ & 35 & $39 \%$ & 45 & $50 \%$ \\
\hline $\begin{array}{l}\text { 2.- ¿Con que frecuencia ha considerado } \\
\text { que los hijos de padres migrantes } \\
\text { tienen mayor riesgo de consumir } \\
\text { drogas? }\end{array}$ & 90 & 41 & $46 \%$ & 30 & $33 \%$ & 19 & $21 \%$ \\
\hline
\end{tabular}




\begin{tabular}{|l|l|l|l|l|l|l|l|}
\hline $\begin{array}{l}\text { 3.- ¿Con qué frecuencia a } \\
\text { considerado que los adolescentes } \\
\text { y/o jóvenes con padres divorciados } \\
\text { tienen mayor riego de consumir } \\
\text { drogas? }\end{array}$ & 90 & 36 & $40 \%$ & 28 & $31 \%$ & 26 & $29 \%$ \\
\hline $\begin{array}{l}\text { 4.- ¿Sufrió discriminación por no } \\
\text { consumir drogas con sus amigos? }\end{array}$ & 90 & 24 & $27 \%$ & 29 & $32 \%$ & 37 & $41 \%$ \\
\hline $\begin{array}{l}\text { 5.- ¿Con qué frecuencia ha considerado } \\
\text { que la presión delos amigos o del } \\
\text { entorno es un factor de riesgo para el } \\
\text { consumo de drogas? }\end{array}$ & 90 & 57 & $63 \%$ & 28 & $31 \%$ & 5 & $6 \%$ \\
\hline $\begin{array}{l}\text { 6.- ¿Con qué frecuencia ha recibido } \\
\text { educación de parte de los docentes y/ o } \\
\text { autoridades sobre la prevención del } \\
\text { consumo de drogas? }\end{array}$ & 90 & 53 & $59 \%$ & 24 & $27 \%$ & 13 & $14 \%$ \\
\hline $\begin{array}{l}\text { 7.- ¿Con qué frecuencia ha recibido } \\
\text { orientación sobre las consecuencias } \\
\text { graves de consumir drogas por parte de } \\
\text { sus padres o familiares? }\end{array}$ & 90 & 21 & $23 \%$ & 30 & $34 \%$ & 39 & $43 \%$ \\
\hline $\begin{array}{l}\text { 8.- ¿Con qué frecuencia ha recibido } \\
\text { educación de parte del personal del } \\
\text { centro de salud (médico, enfermera, } \\
\text { psicólogo, otros) sobre la prevención } \\
\text { de consumo de drogas? }\end{array}$ & 90 & 37 & $41 \%$ & 29 & $32 \%$ & 24 & $27 \%$ \\
\hline $\begin{array}{l}9 .- \text { ¿Obtuvo información sobre la } \\
\text { prevención del consumo de drogas } \\
\text { en la televisión, internet (redes } \\
\text { sociales? }\end{array}$ & 90 & 15 & $17 \%$ & 36 & $40 \%$ & 39 & $43 \%$ \\
\hline $\begin{array}{l}\text { 10.- ¿Obtuvo información sobre la } \\
\text { prevención del consumo de drogas } \\
\text { de sus amigos? }\end{array}$ & 90 & 7 & $8 \%$ & 15 & $17 \%$ & 68 & $75 \%$ \\
\hline
\end{tabular}

Fuente de Elaboración propia

\section{Se presenta en la tabla1, el análisis descriptivo de los datos de la investigación, en la que se}

\section{pudo constatar que:}

El 50\% de los estudiantes de la universidad de Guayaquil consideran que nunca han sabido que algún familiar suyo consumió o consume drogas, mientras el otro $50 \%$ de los estudiantes de la escuela de enfermería han sabido que con frecuencia algún familiar suyo consumió o consume drogas. 
El $46 \%$ según los estudiantes consideran que los hijos de padres migrantes siempre tendrán un mayor riesgo de consumir drogas, mientras que el otro $54 \%$ de los estudiantes creen que a veces o nunca podrían tener el riesgo de llegar a consumir drogas los hijos de padres migrantes.

El $40 \%$ de los estudiantes aprueban que siempre se ha considerado que los adolescentes con padres divorciados tienen mayor riesgo de consumir drogas, mientras tanto el otro $60 \%$ de los estudiantes refieren que a veces o nunca se ha considerado que los adolescentes o jóvenes de padres divorciados tienen mayor riesgo de consumir drogas.

El $41 \%$ de los estudiantes refieren que nunca han sufrido algún tipo de discriminación por no consumir drogas, mientras el 59\% de los estudiantes si han sufrido algún tipo de discriminación por no consumir drogas.

El 63\% de los estudiantes han considerado que la presión de los amigos o de su entorno es un factor de riesgo para el consumo de drogas, mientras $37 \%$ evidencia que a veces o nunca se ha considerado que la presión de amigos o del entorno es un factor de riesgo para el consumo de drogas.

El $59 \%$ de los estudiantes han recibido educación de parte de docentes sobre la prevención del consumo de drogas, mientras el $41 \%$ de los estudiantes refieren que a veces o nunca han recibido educación sobre la prevención del consumo de drogas.

El $43 \%$ de los estudiantes nunca han recibido orientación sobre las consecuencias graves de consumir drogas por parte de familiares, mientras tanto el $57 \%$ de los estudiantes a veces o siempre han recibido orientación sobre las consecuencias de consumir drogas por parte de sus familiares.

El $41 \%$ de estudiantes siempre han recibido educación de parte del personal del centro de salud sobre prevención del consumo de drogas, mientras el $59 \%$ refieren que a veces o nunca han recibido educación sobre prevención del consumo de drogas

El 43\% de los estudiantes nunca obtuvo información de la prevención del consumo de drogas en las redes sociales, mientras el $57 \%$ de los estudiantes dicen que siempre o a veces obtuvieron información sobre la prevención del consumo de drogas por las redes sociales. 
El $75 \%$ de los estudiantes nunca obtuvieron información sobre la prevención del consumo de drogas de sus amigos, mientras el $26 \%$ de los estudiantes siempre o a veces obtuvieron información sobre la prevención del consumo de drogas por parte de sus amigos.

\section{Conclusiones}

Se pudo identificar que el $63 \%$ de la población considera que de los diversos factores el que impacta más sobre el consumo la droga es el factor social el cual siempre está en constante exposición hacia los individuos más susceptibles que ceden a la presión de otras personas para el consumo de ciertas sustancias.

Se reconoció que entre los factores sociales por parte del entorno familiar con un $46 \%$ de los hijos de padres migrantes tiene un mayor riesgo del consumo de drogas por no tener un autocontrol y al no tener una autoridad familiar de primer grado cercana que les pueda informar, guiar sobre que son las drogas. De la misma manera los hijos de padres divorciados con un $40 \%$ siempre son más susceptible al consumo de sustancias estupefaciente.

Se determinó que un $41 \%$ ha recibido una charla educativa sobre drogas por parte de autoridades de salud y otras entidades que son peritos en prevención, el cual contrarresta por parte del entorno familiar el cual nunca con $43 \%$ recibe orientación por partes de padres sobre el consumo de drogas.

Se realizó la participación por parte del personal de internas de enfermería de la Universidad Estatal de Guayaquil en la prevención de consumo de drogas el cual se implementó varias charlas educativas en el alma mater con una buena aceptación por los estudiantes universitarios.

Se determinó que el universo conformado por 90 personas las cuales se pudo comprobar que casi son pocas las personas que toman ciertas medidas preventivas porque no presentan el conocimiento suficiente sobre que es prevención en drogas o de alguna u otra manera piensan que pueden evitar el consumo de estas sustancias, por eso mejor estar informado sobre este tema que afecta a la familia y al entorno psicosocial.

\section{Referencias Bibliográficas}

Asensio, S., Zarco, J., Villanueva, M., y Caudevilla, F. (2012). Programas de prevención del consumo de drogas en el medio escolar: Actividades con profesionales sanitarios. 
España. Recuperado en: http://www.e- drogas.es/c/document_library/get_file?uuid=f4124c5b3b4a-456e-bdec- 66749892eb48\&groupId=10156.

Barros, M. (2013). Efectos del Consumo de drogas sobre la salud de los adolescentes.

Recuperado en: https://repositorio.unican.es/xmlui/bitstream/handle/10902/2992/BarrosBlancoM A.pdf?sequence $=1$.

Careaga Eguía. (2014). Dirección de Salud Pública y Adicciones del Departamento de Salud del Gobierno Vasco y el SIIS Centro de Documentación y Estudios de la Fundación. Desigualdades socioeconómicas, consumo de drogas y territorio. http://www.osakidetza.

Enciclopedia de la Familia. (2011). adolescencia Rehabilitación escuela para Padres. Peú: Tomo 14.

Escohotado, A. (2014). Aprendiendo de las drogas. Usos y abusos, prejuicios y desafíos. Washinton D.C: Digitalizado por libertad http://bit.ly/1dPGBfD.

Hidalgo, M., Redondo, A., Barrio, P., \& Huertas, C. (2013). Consumo de drogas Madrid: Recuperado en: http://cursos.aiu.edu/Desarrollo\%20Humano\%20II\%20Adolescencia/PDF/Tema \%206.pdf.

Instituto de Neurociencias. (2015). El Instituto de Neurociencias en constante lucha contra las drogas. Guayaquil, Ecuador: Junta de Beneficencia de Guayaquil https://www.institutoneurociencias.med.ec/noticias/13- noticia/1409-dia-lucha-contra-las-drogasinstituto-neurociencias-guayaquil.

Instituto Nacional sobre Abuso de Drogas. (2012). La heroína: Abuso y adicción. http://www.drugabuse.gov/es/publicaciones/serie-de-reportes/laheroina-abuso-yaddicion/cuales-son-los-efectos-largo-plazo-del-uso-de-la- heroína.

Instituto Nacional sobre el Abuso de Drogas. (2014). La Heroína: Abuso y adicción. Estados Unidos: http://www.fq.uh.cu/descargas/heroina.pdf.

López, S., Rodríguez, J., \& Arias, P. (2013). Factores de riesgo y de protección en el consumo de drogas y la conducta antisocial en adolescentes y jóvenes españoles. Coruña, España: https://www.google.com.ec/url?sa=t\&rct=j\&q=\&esrc=s\&source=web\&cd=17\&cad=rja\&uact=8 \&ved=0ahUKEwjez5zBhPTRAhUG8CYKHWAOBMM4ChAWCDowBg\&url=https\%3A\%2F 
$\% 2$ Fdialnet.unirioja.es\%2Fdescarga\%2Farticulo\%2F39

74485.pdf\&usg=AFQjCNF4J-

_IHY6Qu5L3NVsufu-d3fqvOw\&bvm=bv.1460.

OSM. (2014). Epidemiología. Cuba: http://www.who.int/topics/epidemiology/es/.

Rimassa, A. (2014). La Droga. Revista vive. Tapad-Criad.: http://www.revistavive.com/la-droga$\mathrm{h} /$.

Secades, R., y Fernández, J. (2012). Consumo de drogas y factores de riesgo en los jóvenes del Municipio de Oviedo. Oviedo, España: http://www.proyectohombreastur.org/fotos/estudio_oviedo.PDF.

UNODC. (2015). Informe mundial sobre las drogas. Viena, Austria: Oficina de las Naciones Unidas contra la Droga y el https://www.unodc.org/documents/wdr2015/WDR15_ExSum_S.pdf.

UNODC. (2015). Informe Mundial sobre las Drogas 2015 encuentra estable el consumo de drogas, y el acceso a tratamiento por Drogas. Viena, Austria: Oficina de Enlace en México. Recuperado en: https://www.unodc.org/mexicoandcentralamerica/es/webstories/2015/informemundial-sobre-las-drogas-2015.html.

UNODC. (2016). Informe Mundial sobre las Drogas. Recuperado en: https://www.unodc.org/documents/wdr2015/WDR15_ExSum_S.pdf.

Whiteford, H. e. (2013). "Carga mundial de morbilidad atribuida a los trastornos mentales y de uso de sustancias: Resultados de la Carga Global de Enfermedad de estudios 2010". The Lancet, vol. 382, no 9.904 , pp. $1575-$

1586. http://www.osakidetza.e. 